\title{
Correlates of Hippocampal Complex-Spike Cell Activity in Rats Performing a Nonspatial Radial Maze Task
}

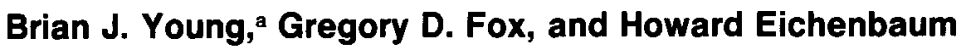 \\ Department of Psychology, University of North Carolina, Chapel Hill, North Carolina 27599
}

\begin{abstract}
The observation of hippocampal place cells forms a major line of evidence supporting the view that the hippocampus is dedicated to spatial processing. However, most studies demonstrating the spatial properties of hippocampal unit activity have employed tasks that emphasize spatial cues but minimize nonspatial cues. In the present experiment we recorded the activity of hippocampal complex-spike cells from rats performing a nonspatial radial maze task. Performance in this task was guided by local visual-tactile cues on the maze arms, while distal spatial cues were minimized and made irrelevant. The influence of three variables on unit activity was examined: type of cue on an arm, spatial location of an arm, and the relative position of the animal on an arm. Of the units recorded, almost one-fifth were classified as "cue cells" in that their activity was associated with cue type but not spatial location. Conversely, a similar proportion of the units were classified as "place cells" in that their activity was associated with location, but not cue type. In an additional similar proportion of units, firing was influenced only by relative position and not by local cues or spatial locations. For the majority of units, however, firing was related to combinations of these three variables, indicating that most hippocampal neurons encoded conjunctions or relations between spatial and local cue information. This pattern of results indicates that when local rather than distal spatial cues are emphasized, hippocampal neural activity is strongly influenced by salient nonspatial cues and shows no overwhelming predominance of place coding. These findings are at odds with the hypothesis that the hippocampus is selectively involved in spatial processing and, conversely, support the broader view that the hippocampus encodes both spatial and nonspatial relations among important experimental variables.
\end{abstract}

[Key words: hippocampus, spatial, nonspatial, single units, CA 1, radial maze, learning, place cells]

In experiments involving the use of an open field or maze apparatus, the activity of hippocampal complex-spike cells often correlates strongly with the location of an animal in the testing

\footnotetext{
Received Dec. 27, 1993; revised Apr. 12, 1994; accepted Apr. 27, 1994.

This work was supported by NIA Grant AG09973. We thank Michael Bunsey, Rebecca Burwell, Tim Otto, Peter Rapp, Mathew Shapiro, and Heikki Tanila for their comments on an earlier version of the manuscript.

Correspondence should be addressed to Howard Eichenbaum, Center for Behavioral Neuroscience, State University of New York at Stony Brook, Stony Brook, NY 11794-2575.

aPresent address: Center for Behavioral Neuroscience, State University of New York at Stony Brook, Stony Brook, NY $11794-2575$.

Copyright (c) 1994 Society for Neuroscience $0270-6474 / 94 / 146553-11 \$ 05.00 / 0$
}

environment (e.g., O'Keefe and Dostrovsky, 1971; O'Keefe, 1976; Olton et al., 1978; Muller and Kubie, 1987). The discovery that most hippocampal complex-spike cells are "place cells" in these situations, combined with a large body of data demonstrating deficits in spatial learning and memory following hippocampal damage, have led to the hypothesis that the hippocampus is selectively involved in processing spatial cues, at least in rats (O'Keefe and Nadel, 1978). Yet despite this impressive evidence indicating hippocampal processing of spatial information, other studies have reported nonspatial firing correlates of the activity of many hippocampal cells in animals performing various simple and discriminative conditioning tasks (Berger et al., 1983; Wible et al., 1986; Eichenbaum et al., 1987; Wiener et al., 1989; Otto and Eichenbaum, 1992; Hampson et al., 1993), casting doubt on the generality of the spatial hypothcsis of hippocampal function.

A more general proposal, arguing that the hippocampus mediates the construction and modification of "relational" memory representations, has been applied to account for both the place cell phenomenon and demonstrations of nonspatial hippocampal firing correlates (Eichenbaum et al., 1992a,b; Cohen and Eichenbaum, 1993). In this conceptualization, the hippocampus encodes critical relations among salient cues and constructs a memory organization of relevant items and events. The encoding of spatial relations in the environment is thus a straightforward example of such relational representation, and indeed is expected to be a major component of hippocampal representation in virtually all situations where the animal perceives and remembers a spatial organization of items and events.

According to this view of hippocampal function, a reconciliation of the discrepant findings regarding functional correlates of hippocampal activity may lic in the differing stimuli and response demands of the different behavioral paradigms that have been employed. In particular, experiments reporting a predominance of place correlates involve environments and behavioral requirements that emphasize prominent distal cues and require subjects to process spatial relations among these cues to predict the locus of rewards. Further, these experiments minimize nonspatial cues and make them irrelevant to successful task performance. For example, O'Keefe and Conway (1978) trained rats to locate food rewards using spatial relationships among a set of prominent distal cues positioned around the perimeter of a screened testing environment. They provided no obvious distinguishing cues among the arms of the maze and rotated the distal spatial cues and the reward site between trials, making the spatial cues, but not any remaining local cues, predictors of reward. Under these conditions, the activity of most hippocampal complex-spike cells reflected the spatial relationships among the distal cues. This finding, along with similar 
results from studies using open-field test environments in which distal cues are emphasized (Olton et al., 1978; Kubie and Ranck, 1983; McNaughton et al., 1983; O'Keefe and Speakman, 1987), supports the conclusion that the hippocampus detects and processes salient spatial relationships in the environment. However, such findings do not exclude the possibility that, under circumstances where spatial cues are not emphasized, hippocampal cells may just as strongly process nonspatial information. Consistent with this expectation, striking nonspatial correlates of hippocampal unit activity have been observed in a variety of formal learning paradigms where spatial cues are minimized or made irrelevant (Berger et al., 1983; Wible et al., 1986; Eichenbaum et al., 1987; Wiener et al., 1989; Otto and Eichenbaum, 1992).

One explanation for the predominance of nonspatial correlates of hippocampal activity in rats performing some tasks, and the predominance of spatial correlates in other tasks, is that hippocampal representations can change to reflect the salience of existing stimuli. Alternatively, the many differences between thc testing procedures and behavioral requirements used in openfield or maze environments, where spatial firing correlates predominate, compared with those used in the simple conditioning and discrimination tasks, where nonspatial correlates predominate, may explain the discrepancies in the findings. To determine whether hippocampal representations do indeed change to reflect the salience and relevance of the available stimuli, it would be valuable to compare the firing correlates of hippocampal cells described in studies where recordings were made in rats performing a spatial maze task with results obtained using a maze task that is altered to reduce the emphasis on spatial cues. In the present study we recorded hippocampal complexspike cell activity from rats performing a nonspatial working memory task on a four-arm radial maze. We selected the paradigm created by Olton and Feustle (1981) because this task involves the same kind of environment and the identical behavioral requirements typically used in spatial radial maze tasks, except that performance is guided by prominent local cues while distal spatial cues are minimized and made irrelevant. Thus, the results obtained in this nonspatial task can be directly compared with those from studies using spatially guided radial maze tasks (Olton et al., 1978; Kubie and Ranck, 1983; McNaughton et al., 1983; O'Keefe and Speakman, 1987). We predicted that, in contrast to the findings of experiments using spatial tasks, a large fraction of hippocampal units would have firing correlates strongly associated with specific local cues. In addition, we did not expect the absence of spatial firing correlates in this task because, despite efforts to minimize the salience and relevance of spatial cues, there remained multiple potential sources of spatial information (see below) and it is likely that a sense of spatial orientation is always relevant to a rat exploring a large open environment. Thus, our expectations were that, under the circumstances of the present study, both nonspatial and spatial cues may be reflected in hippocampal unit firing correlates.

\section{Materials and Methods}

Subjects. Five male Long-Evans rats weighing between 350 and 400 $\mathrm{gm}$ at the beginning of the experiment served as subjects. The animals were individually housed, maintained on a 12:12 hr light:dark cycle, and given ad libilum access to water. Food was restricted to maintain body weight at $90 \%$ of the free-feeding weight with gains of approximately 5 gm every 2 weeks.

Electrodes and surgery. All subjects were pretrained on the maze task outlined below prior to electrode implantation. The electrode assemblies consisted of ten $25 \mu \mathrm{m}$ Formvar-coated nichrome wires of equal lengths bundled into a 26 gauge cannula (Eichenbaum et al., 1977) and attached to a vertically driveable connector (Kubie, 1984). The animals were anesthetized with sodium pentobarbital $(50 \mathrm{mg} / \mathrm{kg}$, i.p.) supplemented with methoxyflurane when necessary, and administered atropine $(0.5$ $\mathrm{mg}$ in $1 \mathrm{cc}$, i.p.) to reduce mucous secretions. Using aseptic surgical procedures, the electrode assemblies were implanted stereotaxically, with the skull level, at the following coordinates: $3.2 \mathrm{~mm}$ posterior to and $1.8 \mathrm{~mm}$ lateral to bregma, and $1-1.5 \mathrm{~mm}$ below the pial surface. The base of the electrode was attached to the surface of the skull using dental cement and four stainless steel screws, one of which was secured to the frontal bone and served as the electrical ground. Following surgery the animals were given ad libitum access to food for a minimum of 3 $\mathrm{d}$ before being placed back on the food deprivation schedule.

Unit recording and data acquisition. The subjects were screened one or two times per day for unit activity. If no activity was identified during screening, the electrode was advanced approximately $40 \mu \mathrm{m}$ and allowed to settle for at least $4 \mathrm{hr}$ prior to subsequent screening. Up to four individual channels of neural activity were passed individually through a high-impedance JFET headstage, then to an AC amplifier (Grass, Model P511K) where the signal was amplified $2000 \times$ and bandpass filtered at $300-3000 \mathrm{~Hz}$. Spike detection was performed using the time and amplitude window discriminators of a computerized data acquisition system (Cambridge Electronic Design, model 1401+). In cases where two or more units were recorded on the same channel, the amplifier output was passed to multiple window discriminators with nonoverlapping thresholds for unit isolation. Only units with signal to noise ratios of at least 3:1 were recorded. To eliminate recording artifacts, a separate "noise" channel with no detectable spike activity was recorded. Window thresholds for this channel were set to the maximum and minimum levels of the unit channels, and the number of noise events recorded in a given region of a maze arm was subtracted from the number of spike events recorded in the same region.

The position of the rat in the maze was determined by a custom-built video camera following system that tracked an incandescent light bulb mounted on the JFET headstage assembly. Location was digitized in the form of $x$ - and $y$-coordinates at a rate of $20 \mathrm{~Hz}$ by A/D converters in the data acquisition system.

Behavioral apparatus. The apparatus and behavioral procedure used in this experiment were modeled after those of Olton and Feustle (1981). The apparatus consisted of a four-arm radial maze elevated $70 \mathrm{~cm}$ above the floor and constructed of black-painted wood and black Plexiglas. Adjacent arms were oriented at $90^{\circ}$ to each other and attached to an octagonal central platform that measured $25 \mathrm{~cm}$ between opposite sides. Each arm was covered by one of four movable arm overlays. The overlays extended the entire length of the arms, measuring $46 \mathrm{~cm}$ long and $9.5 \mathrm{~cm}$ wide, and had sides that were $2.5 \mathrm{~cm}$ high. Except for a $5-\mathrm{cm}$ long black-painted area at the end where a recessed food cup was located, the floor and sides of each overlay were covered with one of four distinctive visual-tactile cues: coarse tan-colored sandpaper (\#36D), black corrugated rubber, fine aluminum screen (approximately $1 \mathrm{~mm} \times 1$ $\mathrm{mm}$ ) on a black-painted background, or coarse black plastic mesh (approximately $5 \mathrm{~mm} \times 5 \mathrm{~mm}$ ) on a gray-painted background. Access to each arm was restricted by $22 \mathrm{~cm}$ high walls, and by remotely controlled reverse-guillotine doors of the same height; these could be raised to restrict the rat to the central platform or lowered to permit the animal to venture onto the arms.

The location on the maze relative to other features of the recording room is diagrammed in Figure 1. In an attempt to minimize extramaze cues, a $185 \mathrm{~cm}$ square area surrounding the maze was screened from the rest of the room by black blackout curtains that extended from the floor to the ceiling. In addition, the floor was covered with black carpeting, the ceiling painted flat black, and white noise ( $70 \mathrm{~dB} \mathrm{SPL}$ ) was delivered through two $10 \mathrm{~cm}$ speakers mounted on the ceiling above the maze. The environment within the curtains was dimly illuminated by four $110 \mathrm{~V}, 50 \mathrm{~W}$ track-light fittings connected to a Variac set at 35 $\mathrm{V}$. The remote controls for the maze and data collection system were located in a consistent place outside the maze; auditory stimuli arising from this apparatus might have provided subtle cues for spatial orientation.

Behavioral procedures. The animals first received $5 \mathrm{~d}$ of preexposure during which they were placed on the maze with the doors open for 10 min per day and were allowed to collect food rewards from the end of each arm. Behavior was observed continuously using a video monitor connected to the tracking system. Following preexposure, the animals were given two sessions of training per day, separated by approximately 


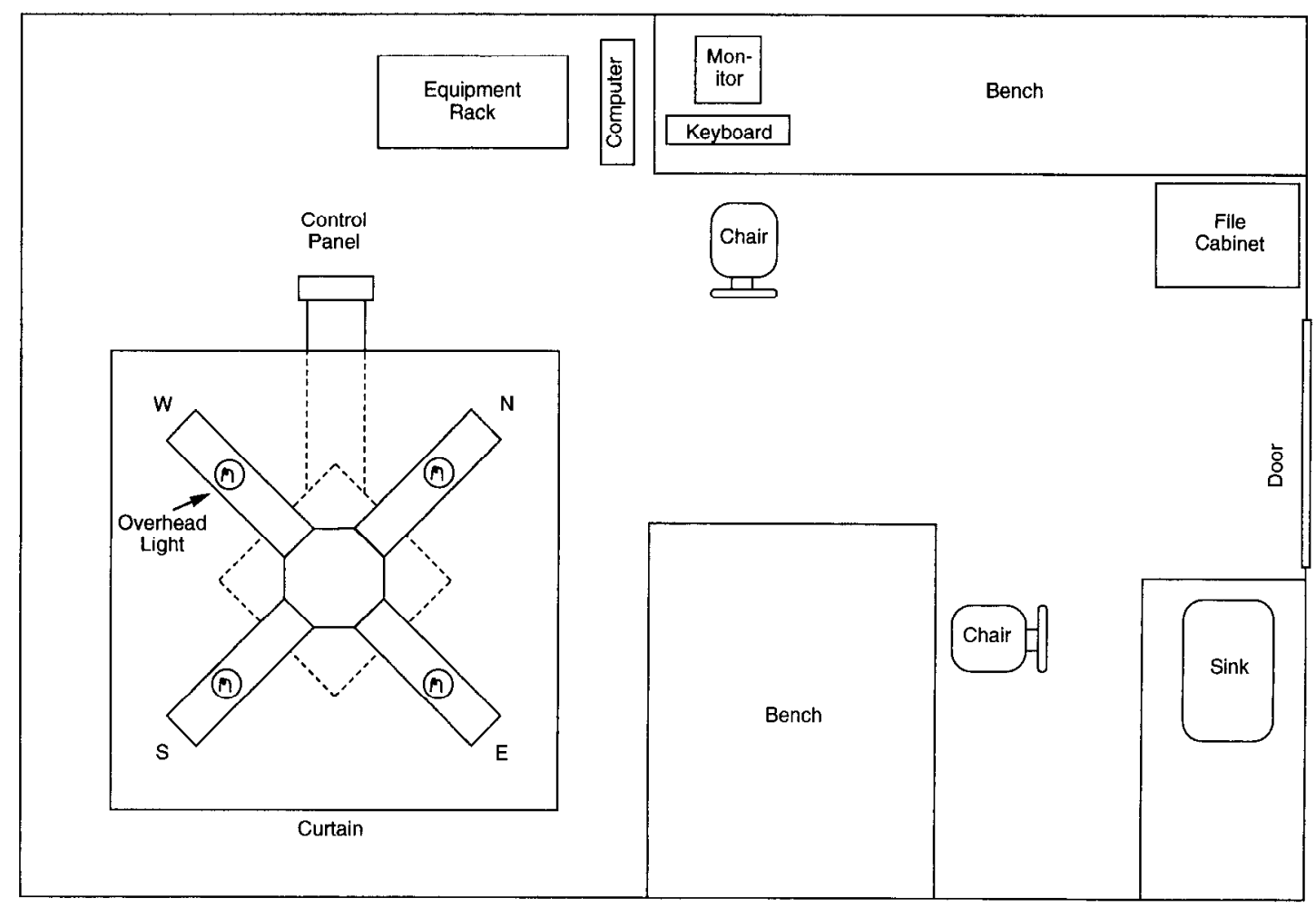

Figure 1. Overhead view of the testing environment.

$5 \mathrm{hr}$. At the beginning of a session, each of the four arms was baited with four $45 \mathrm{mg}$ Noyes pellets (Formula A), the rat was placed on the center platform with the doors closed, and the experimenter left the screened area. A trial was initiated when the experimenter simultaneously opened all four doors from the control panel. When the rat placed all four paws on an arm, the experimenter closed the doors of the three remaining arms and then waited for the animal to return to the center platform before closing the remaining door. During the intertrial interval the experimenter entered the screened area from one of the four corners and changed the topological arrangement of the arm inserts, moving the remaining food pellets along with the inserts but not rebaiting previously selected choices. The arrangement of arms on each trial was determined from a list of the 24 possible arm combinations sequenced in a pseudorandom order. An example of arm arrangements used on four successive trials is shown in Figure 2. The corner of the screened area where the experimenter entered was also varied pseudorandomly.

Each session lasted for as many trials as required for the animal to retrieve the four baits up to a maximum of 10 trials. Perfect performance required a rat retrieve all of the baits in four successive trials, each involving a visit to an arm identified only by a unique cue overlay. Training continued until each animal had attained a mean performance criterion of 3.8 correct responses in the first four trials of each session averaged across 10 successive sessions, the same performance criterion used by Olton and Feustle (1981) in their experiment demonstrating the critical involvement of the hippocampal system for performance of this task.

Having reached criterion, the animals were implanted with the chronic recording electrodes and given at least $5 \mathrm{~d}$ to recover. During the recording phase, behavioral procedures were fundamentally the same as those described above, except that multiple sessions were run to provide sufficient data for statistical analysis, and a unit screening period was added. During the screening period, the animal was allowed to move freely around the maze with all doors open. No baits were on the maze during this time. Following the setting of spike discrimination parameters, 6-12 consecutive sessions were run, using the procedures described above. Due to the number of trials run during these recording sessions, the size of the food baits was reduced to two $45 \mathrm{mg}$ pellets. Also, in an effort to reduce the possible interference effects of previous sessions, animals were removed from the maze for 10 min between sessions and placed on top of the maze in a galvanized steel tub with a floor covered in wood chips. When more than eight consecutive sessions were run, subsequent sessions involved the use of forced trials. On forced trials, only doors to baited arms were opened. The doors were opened one at a time in a pseudorandom sequence until the animal had retrieved all four rewards, with successive doors being opened after the animal had returned to the maze center.

Data analysis. The principal goal of the analysis was to determine the extent to which complex-spike cell activity was associated with the distinctive local cues that guided performance in the task, as wcll as with spatial location. To accomplish this we assessed unit firing rates associated with the four cue types in each of the four locations. Additionally, the relative position of the animal on an arm, independent of the presented cue or spatial location, was also entered into the analysis. Relative position was determined by dividing each arm into four equalsized regions radiating outward from the center platform. The four positions for each arm are represented in Figure 3 by the broken lines superimposed on an outline diagram of the maze. Unit activity was calculated on a given trial by dividing the number of spikes recorded within each position by the amount of time (in seconds) spent in the region associated with that relative position.

The firing patterns of the units were analyzed using two different methods. First, the mean firing rates associated with each relative position were plotted on four schematic diagrams of the mazc vicwed from overhead. Each diagram represents a particular cue in each of the four locations, with North oriented toward the top. Further, the individual arms on each diagram were divided into four regions to represent relative position. Thus, means are presented for all possible combinations of the four cues, four spatial locations, and four relative positions. As an extension of the firing rate plots, single-ended confidence intervals were computed for the activity of cells at each "pixel" in the firing rate plot. Pixels associated with a firing rate at least 1.65 SDs above the mean for all pixels were considered to reflect significant local increases in firing rate. This method was employed to identify specific areas of increased unit activity on firing rate plots (see Figs. 4-7), and was not intended for definitive comparisons of the influence of cue, spatial, and relative position variables. Second, to compare directly the extent to which cellular activity was determined by the variables under investigation, units for which sufficient data were obtained were subjected to an analysis of variance involving four levels for each of the three factors, that is, for four cue types (CT), four spatial locations (SL), and four relative positions on the goal arm (RP). For units where no more than 


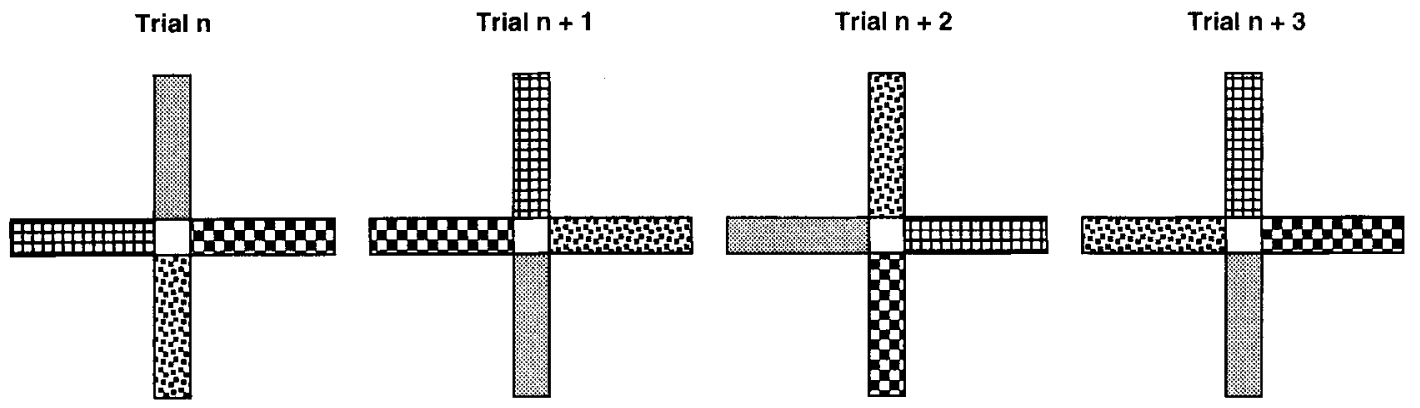

Figure 2. Example of the arm arrangements used for four consecutive trials. The four different patterns represent the distinctive visual-tactile arm overlays that were used as local cues.

one trial was run for one or more cue-location combinations, a twoway analysis incorporating only the CT and SL factors was used. Trial effects were not considered; the data were collapsed across trials in both types of analysis. Only trials on which the animal entered a rewarded arm were analyzed. Since no systematic differences were observed between the patterns of activity of forced and regular trials, both types of trials were analyzed together. Effects with a $p<0.05$ were regarded as significant. Finally, to evaluate the extent to which these variables controlled the firing pattern of each neuron, an estimate of the proportion of variance accounted for by each significant main effect and interaction was calculated.

\section{Results}

\section{Behavioral performance}

The mean number of sessions required to reach the performance criterion was 62.2 (range, 26-97). Performance decreased somewhat during the recording sessions from the 3.8 training criterion to an overall mean of 3.43 correct responses in the first four trials of each session. This decrease, representing a decline in performance from $95 \%$ to $86 \%$ correct, likely reflects interference effects associated with the presentation of multiplc consecutive sessions during the recording phase of the experiment.

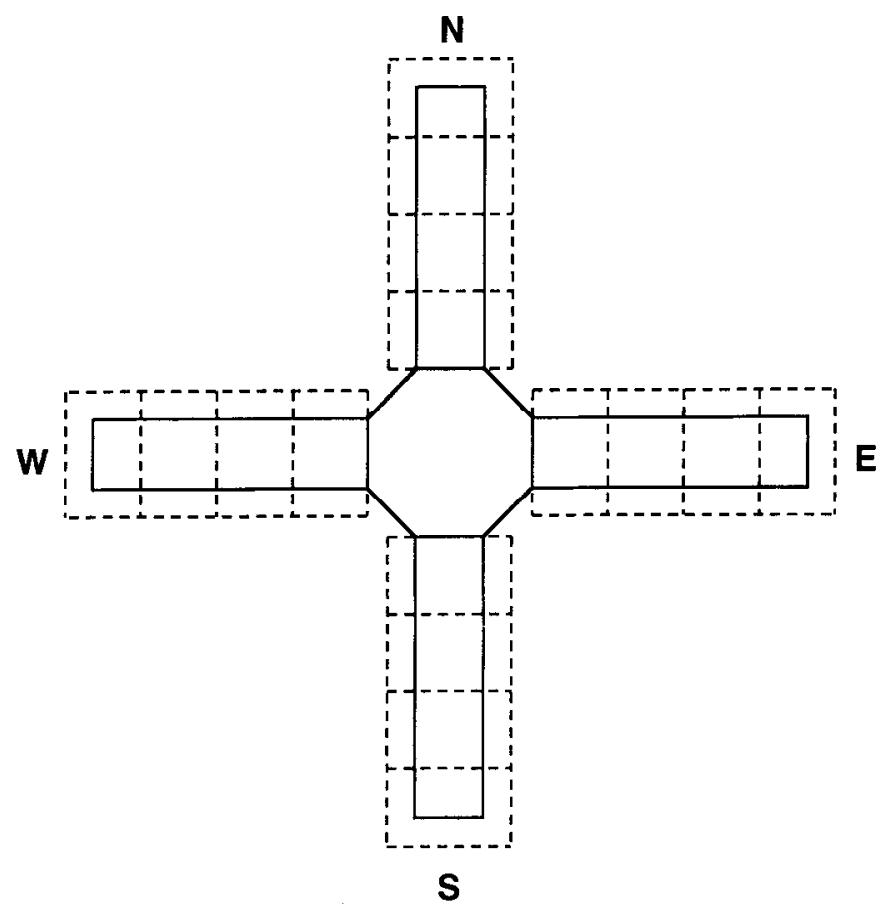

Figure 3. The regions used to delineate the four relative positions of each arm.

\section{Physiological data}

A total of 90 units identified physiologically as complex-spike cells (Ranck, 1973) were recorded from areas CAl (66 units; $73.3 \%$ ) and CA3 (24 units; $26.7 \%$ ) of the hippocampus. As there were no systematic differences in the firing correlates of $\mathrm{CA} 1$ and CA3 units, no further distinction is made between units from the two areas. Sufficient data were collected to perform three-factor ANOVAs on 58 units, with the remaining 32 units being analyzed with two-factor ANOVAs. Of those units subjected to the three-factor analysis, significant associations with at least one of the three factors were obtained for $49(84.5 \%)$ of the units (Table 1); however, most cells displayed activity that was associated with at least two of the three factors. Thirty-two of the 49 units $(65.3 \%)$ had firing increases associated with the type of cue, $30(61.2 \%)$ had firing increases associated with spatial location, and $41(83.7 \%)$ had activity increases associated with relative position. Of those units analyzed using the twofactor ANOVA, 20 (62.5\%) had significant firing correlates (Table 2$)$. The activity of 12 of these units $(60.0 \%)$ was associated with CT and the activity of 17 units $(85.0 \%)$ was associated with SL. In addition, consistent with previous findings on hippocampal complex-spike cells recorded in freely moving rats performing tasks that involve spatially oriented movements, some of the cells in the present study displayed firing that was associated with the animal's direction of locomotion (McNaughton et al., 1983; Wiener et al., 1989). For example, the cell displayed in Figure 7 exhibited increased activity only when the rat was traveling toward the maze center. While directionality was observed for a substantial proportion of cells, it is not strictly relevant to the hypothesis under investigation in the present study, and hence is not considered further in this analysis.

Our subsequent considerations of these findings focused on the comparison of cue versus spatial factors in controlling cell activity and the interpretation of relative position as an important variable in determining cell firing. With regard to the latter issue, firing associated with relative position on a maze arm could reflect at least three distinct properties by which this factor either qualifies the spatial locations and/or cue type variable(s), or represents an independent influence over firing. First, for cells with activity associated with spatial location, a significant effect of RP likely reflects a restricted region of increased firing within a maze arm, as typically reported for "place fields." Thus, a cell with activity demonstrating a significant SL factor, with or without a significant interaction between the SL and RP factors, and a nonsignificant CT factor can be considered a "place cell." Second, following a parallel logic for cells with activity associated with cue type, relative position may corre- 
Table 1. Percentage of the variance accounted for by the significant main effects and/or interactions obtained in the three-way ANOVAs

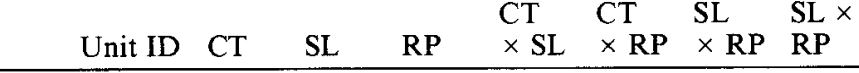

\begin{tabular}{|c|c|c|c|c|c|c|c|c|}
\hline 1 & $507-1$ & 6.7 & & & & & & \\
\hline 2 & $513-3$ & 5.2 & & & & & & \\
\hline 3 & $502-2$ & 14.4 & & 5.7 & & & & \\
\hline 4 & $504-3$ & 7.0 & & 11.8 & & & & \\
\hline 5 & $505-3$ & 6.6 & & 27.3 & & & & \\
\hline 6 & $508-1$ & 7.4 & & 18.6 & & & & \\
\hline 7 & $608-3$ & 5.4 & & 6.5 & & & & \\
\hline 8 & $511-1$ & 6.7 & & 6.9 & & 7.9 & & \\
\hline 9 & $511-3$ & 13.2 & & 7.4 & & 17.9 & & \\
\hline 10 & $604-2$ & 6.7 & & 13.6 & & 8.5 & & \\
\hline 11 & $503-1$ & & 8.1 & & & & & \\
\hline 12 & $506-2$ & & 13.4 & & & & 12.2 & \\
\hline 13 & $503-3$ & & 6.9 & 15.1 & & & 8.2 & \\
\hline 14 & $506-1$ & & 9.7 & 5.0 & & & 9.3 & \\
\hline 15 & $509-1$ & & 8.0 & 15.1 & & & 23.8 & \\
\hline 16 & 1003-2 & & & 17.6 & & & 15.3 & \\
\hline 17 & $501-2$ & & & 8.8 & & & 9.7 & \\
\hline 18 & $605-1$ & & & 10.7 & & & 10.3 & \\
\hline 19 & $501-1$ & & & 4.8 & & & & \\
\hline 20 & $504-1$ & & & 6.3 & & & & \\
\hline 21 & $504-2$ & & & 7.8 & & & & \\
\hline 22 & $508-3$ & & & 13.5 & & & & \\
\hline 23 & $509-2$ & & & 8.2 & & & & \\
\hline 24 & $602-1$ & & & 13.9 & & & & \\
\hline 25 & $603-1$ & & & 7.7 & & & & \\
\hline 26 & $608-1$ & & & 5.7 & & & & \\
\hline 27 & $608-2$ & & & 10.3 & & & & \\
\hline 28 & $1003-1$ & 8.0 & 10.3 & & & & & \\
\hline 29 & $502-3$ & & & & 24.3 & & & \\
\hline 30 & $505-1$ & & & & 17.5 & & & \\
\hline 31 & $508-2$ & & & & 11.6 & & & \\
\hline 32 & $512-1$ & & & & 12.5 & & & \\
\hline 33 & $607-1$ & & & 4.4 & 8.4 & & & \\
\hline 34 & $510-1$ & 4.1 & & 9.5 & 8.0 & & & \\
\hline 35 & $606-3$ & & 14.5 & 15.0 & & 10.7 & & \\
\hline 36 & $512-2$ & 7.2 & & & 12.1 & 7.8 & & \\
\hline 37 & $506-3$ & & & 11.6 & & 9.3 & 7.0 & \\
\hline 38 & $503-2$ & & 4.5 & 13.0 & & 7.4 & 9.6 & \\
\hline 39 & $513-2$ & & 9.2 & 5.7 & 10.0 & & 10.4 & \\
\hline 40 & $607-2$ & & 3.8 & 17.0 & 8.3 & & 8.0 & \\
\hline 41 & $1004-1$ & & 12.9 & 12.1 & 8.4 & & 12.7 & \\
\hline 42 & $1001-1$ & 3.5 & 9.1 & 5.9 & 22.0 & & 12.4 & \\
\hline 43 & $505-2$ & 3.7 & & 17.7 & & & & 21.7 \\
\hline 44 & $510-2$ & 2.8 & 4.1 & 3.6 & & 16.8 & & 20.2 \\
\hline 45 & $507-2$ & & 2.9 & 28.4 & 5.8 & & 7.1 & 15.7 \\
\hline 46 & $512-3$ & 2.1 & 12.6 & 4.1 & 4.8 & 7.1 & 18.0 & 16.9 \\
\hline 47 & $1005-1$ & 5.6 & 6.5 & 4.5 & 11.0 & & 8.5 & 18.2 \\
\hline 48 & $513-1$ & 6.6 & 7.4 & 2.9 & 13.0 & & 10.7 & 17.4 \\
\hline 49 & $1005-2$ & 6.7 & 4.4 & 4.6 & 17.5 & 6.1 & 5.1 & 23.1 \\
\hline
\end{tabular}

spondingly reflect specificity of firing associated with a distinctive part of the cue, for example, the goal end of the sandpaper arm. Thus a cell with activity demonstrating a significant CT factor, with or without a significant interaction between the CT and RP factors, and a nonsignificant SL factor was considered
Table 2. Percentage of the variance accounted for by the significant main effects and/or interactions obtained in the two-way ANOVAs

\begin{tabular}{rrrrr} 
& Unit ID & CT & SL & CT $\times$ SL \\
\hline 1 & $407-1$ & 22.6 & & \\
2 & $412-2$ & 9.8 & & \\
3 & $1002-1$ & 8.3 & & \\
4 & $204-2$ & & 12.5 & \\
5 & $404-1$ & & 9.6 & \\
6 & $406-1$ & & 11.7 & \\
7 & $415-1$ & & 11.4 & \\
8 & $416-1$ & & 14.5 & \\
9 & $416-2$ & & 23.8 & \\
10 & $417-1$ & & 10.5 & \\
11 & $417-2$ & & 25.7 & \\
12 & $413-2$ & 10.7 & 15.1 & \\
13 & $415-2$ & 11.2 & 20.5 & \\
14 & $202-1$ & & & 18.9 \\
15 & $208-1$ & & & 11.1 \\
16 & $209-1$ & & & 16.1 \\
17 & $601-1$ & & & 15.2 \\
18 & $205-3$ & & 11.4 & 19.2 \\
19 & $408-1$ & 9.6 & 10.5 & 15.0 \\
20 & $418-2$ & 10.0 & 11.1 & 24.7 \\
\hline
\end{tabular}

a "cue cell." Third, for cells with no significantly elevated firing associated with either cue type or spatial location increased firing associated with relative position may reflect either a particular behavior that reliably occurs in one part of the arm, for example, sniffing at the ends of arms, or a relative spatial parameter associated with the radial distance of the rat from the central platform. Cells with activity demonstrating a significant RP factor and no other significant factor or interaction were considered to reflect relative position per se. A consideration of the proportions of these cell types and examples of each of these and their combinations follows.

\section{Unit activity associated with cue type and not spatial location}

Of a total of 69 units subjected to the three- or two-way ANOVAs, the activity of 13 units (18.8\%) was significantly related to the CT factor, with or without a significant RP factor, but not to the SL factor (Table 1, units 1-10; Table 2, units 1-3). These cells can be considered "cue cells" in that their activity reflected the type of cue on the arm when the rat was present, with no significant influence of the spatial position of the arm. The proportion of the total variance accounted for by significant $\mathrm{CT}$ and $\mathrm{CT} \times \mathrm{RP}$ effects ranged between $2.1 \%$ and $22.6 \%$, with a mean of $9.6 \%$.

The pattern of firing rates throughout the maze and indications of areas of significantly elevated firing rates for one such unit are presented in Figure 4. This cell displayed striking and significantly elevated firing when the rat was on arms with cue 4 , and no elevation of firing when that cue was not present, resulting in a significant main effect of CT $[F(3,191)=10.31$, $p<0.001]$. Furthermore, this cell fired only when the rat was near the middle of cue $4 \mathrm{arms}$, as indicated by the firing rate plot and confirmed by the significant CT $\times$ RP interaction $[F(9,191)=4.68, p<0.001]$. Although there was no significant main effect for SL, nor any significant interaction involving SL, the activity of this cell might have been influenced to a minor 


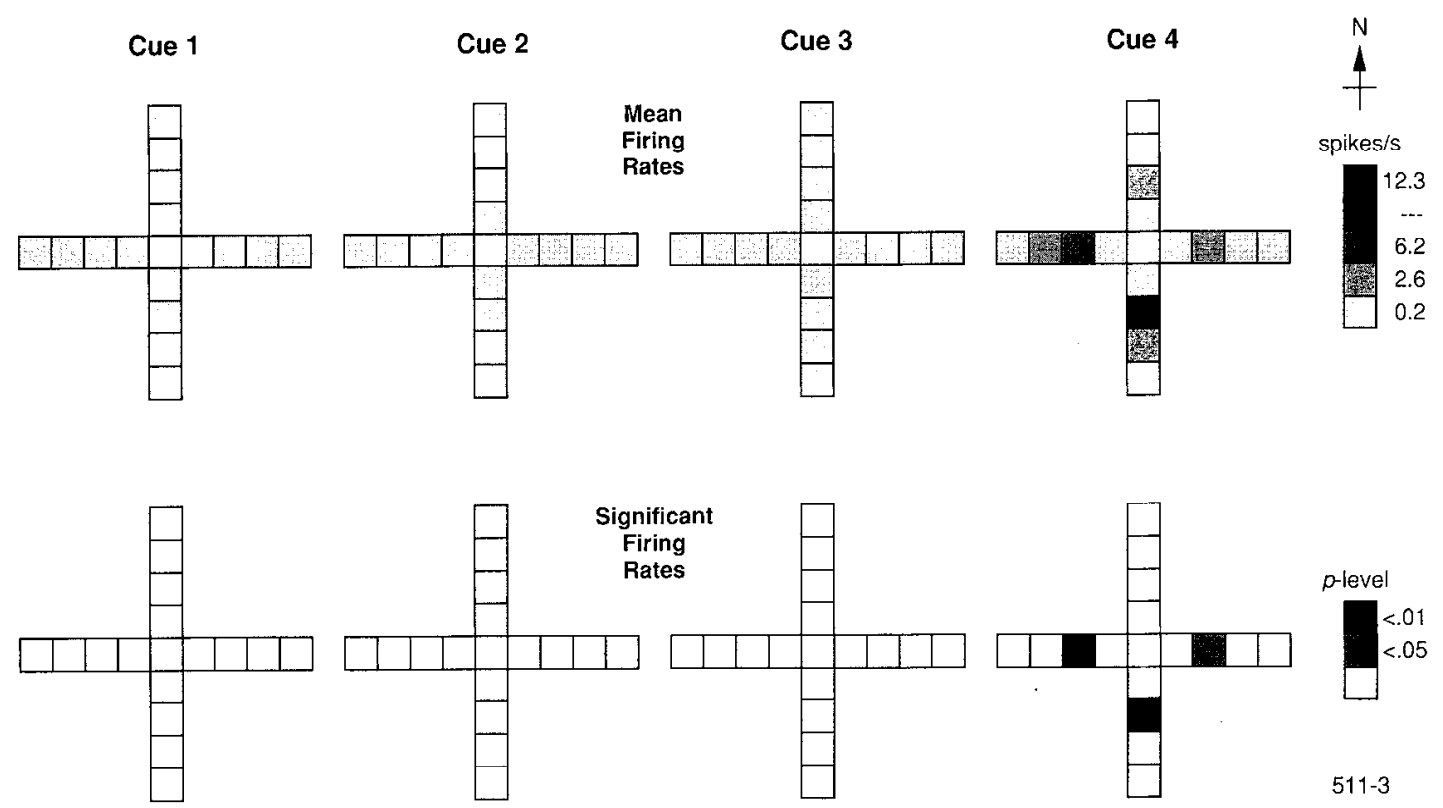

Figure 4. Example of a "cue cell." The top row shows the mean firing rates calculated for each relative position of each cue in each of the North, South, East, and West locations. Firing levels assigned to the shadings in the legend were determined by dividing the maximum firing rate by five to produce five equal ranges. The median firing rate for each range was then calculated and assigned to the corresponding shading. When no relative position had a mean firing rate that fell within a particular range, a dashed line was assigned to its corresponding shading. Significant local changes in firing rate are displayed in the bottom row. Firing rates of relative positions that were greater than 1.65 SDs above the mean, but less than or equal to 2.33 SDs above the mean, were designated as $p<0.05$, representing their significance level. Firing rates greater than 2.33 SDs above the mean were designated as $p<0.01$. Figures 5-7 are presented in an identical manner.

extent by spatial location. The firing rate plots indicate significant local changes in firing rate only when the rat was on cue 4 in the East, South, or West positions, but not on the North position (Fig. 4). Nevertheless, as the upper panel of Figure 4 shows, activity was apparently elevated to some extent in the same relative position of the North arm as that of the other arms. Given that the ANOVA is the more direct and conservative analysis of the relative effects of the factors tested here, our conclusion is that the activity of this cell and all cells in this category strongly reflect the relevant specific cues and are poorly, if at all, influenced by spatial location.

\section{Unit activity associated with spatial location and not cue type}

The activity of 16 of the 69 units (23.2\%) subjected to two- or three-way ANOVAs was significantly related to the SL factor, with or without a significant RP factor, but not to the CT factor (Table 1, units 11-18; Table 2, units 4-11). These cells can be considered "place cells" in that their activity reflected the spatial position of the arm in which the rat was present, with no significant influence of the type of cue on the arm. The proportion of the total variance accounted for by significant SL and SL $\times$ RP effects ranged between $6.9 \%$ and $25.7 \%$, with a mean of $12.73 \%$.

The firing rate plot for a unit that demonstrated locationrelated firing in the absence of cue-related firing is shown in Figure 5 . The activity of this unit was characterized by increased firing when the rat was on arms in the South location, and the firing increase was further restricted to the region of the arm closest to the central platform. Correspondingly, the ANOVA revealed both SL and RP main effects $[F(3,191)=7.42, p<$ 0.001 , and $F(3,191)=14.00, p<0.001$, respectively], as well as an SL $\times \mathrm{RP}$ interaction $[F(9,191)=7.34, p<0.001]$. Although this analysis reveals no significant main effect or inter- action with cue type, the firing rate plots suggest that the activity of this cell might have been influenced to a minor extent by the cues. This plot shows a region of significantly increased firing when the rat was in the West location when cue 1 was present, and that significantly increased firing did not occur when the rat was on the South arm if cue 2 was present. While this effect of CT was quite striking in the firing rate plot, it was not detected by the ANOVA; therefore, we conclude that for this cell, and for all cells in this category, spatial location was the primary determinant of firing and activity was poorly, if at all, influenced by the local cues.

\section{Unit activity associated with relative position}

As stated above, the activity of many units that was related to the CT or SL factors was also related to the RP factor. Significant elevations in firing associated with the RP factor were observed for 41 units $(83.7 \%)$ of the total of 49 subjected to the threeway ANOVAs, which included an analysis of this factor (Table 1 , units 3-10, 12-27, 33-49). Of these, the activity of 32 units was also related to cue type and/or spatial location. In such cases, we interpret the significant RP factor as a qualification or specification of the cue or location represented. However, the activity of the remaining nine units $(18.4 \%)$ was associated with the RP factor in the absence of any significant association with CT or SL (Table 1, units 19-27), indicating that relative position per se is encoded independently, at least by some hippocampal cells, in this task. The proportion of the total variance accounted for by the RP effect for these nine cells ranged between $4.8 \%$ and $17.6 \%$, with a mean of $8.7 \%$.

An example of a unit whose activity was significantly influenced only by relative position, reflected in a main effect of RP $[F(3,183)=4.96, p<0.003]$, is shown in Figure 6. Although the ANOVA revealed no significant CT or SL effects, there might 

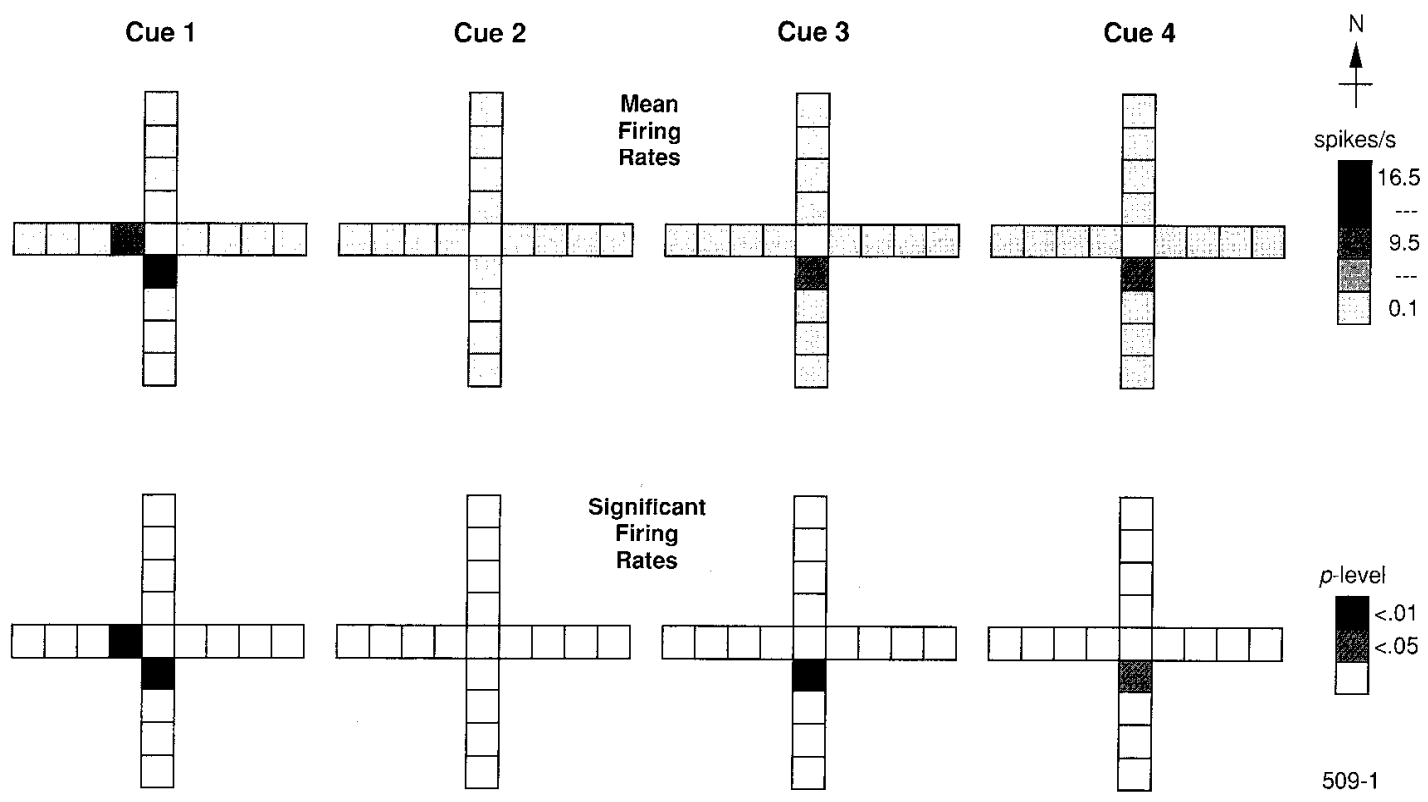

Figure 5. Example of a "place cell."

have been some influence of these factors. Examination of the firing rate plots show that, with the exception of cue 3 , the firing rate of the unit is elevated in the third position from the central platform for all cue and location combinations. In the case of cue 3 , activity in this position increased only when the cue was in the North location. Significant local changes in firing rate were found only in the third position and, as Figure 6 shows, only in four cue-location combinations. Nevertheless, because the ANOVA detected only the RP effects, our conclusion is that the activity of this cell, and all cells in this category, is influenced significantly only by relative position.

\section{Interactions between cue type, spatial location, and relative position factors}

As stated above, the activity of most of the 49 units in the threeway analysis and 20 units in the two-way analysis for which significant effects were obtained was related to more than one factor. Twenty-nine units $(42.0 \%)$ of the total from both types of analysis displayed activity that was associated with two of the factors (Table 1, units 3-10, 12-18, 28-32; Table 2, units $12-20$ ), and the activity of a further 17 units (34.7\%) of the 49 from the three-way analysis was associated with all three factors (Table 1, units 33-49). Of these 46 units (66.7\%) whose activity was significantly associated with either two or three factors, the activity of $31(67.4 \%)$ was significantly related to the CT and SL factors. As much as $67.5 \%$ of the total variance of firing for an individual cell (Table 1, unit 49) could be accounted for by summing the contributions of the individual factors and their interactions.

A striking example of an interaction between all three factors is shown in Figure 7. Reliable increases in the activity of this unit were seen only when the rat was in the North location, cue 3 was present, and the animal was approximately equidistant between the end of the arm and the central platform. Correspondingly, the ANOVA revealed a three-way interaction $[F(27,191)=3.63, p<0.001]$, as well as significant two-way interactions $[F \mathrm{~S}(9,191)>2.2, p \mathrm{~s}<0.03]$ and significant main effects for each factor $[F \mathrm{~s}(3,191)>5.72, p \mathrm{~s}<0.001]$.

\section{Discussion}

In the present study rats were required to perform a working memory task in which accurate performance relied on the use of local, nonspatial visual-tactile cues. The results confirm our two principal experimental predictions. First, the activity of a majority of hippocampal complex-spike cells was significantly influenced by specific local cues and, for a substantial proportion of these cells, firing was not associated with the spatial location of the animal. Second, the activity of the majority of hippocampal cells was also influenced by spatial location, with the firing of a substantial subset of these cells unrelated to the nonspatial cues. In addition, the relative position of the animal on an arm, independent of either cue or location, also emerged as an important determinant of hippocampal unit activity, indicating that either relative position itself, or a behavior that consistently occurs at particular relative positions, is also related to hippocampal unit activity in rats performing this task. Furthermore, the proportions of cells whosc firing reflected specific cues but not spatial position ("cue cells"), cells whose firing reflected spatial location correlate but not local cues ("place cells"), and cells whose firing reflected only relative position were approximately equivalent, indicating each of these factors was an important component of hippocampal representation in this task. Finally, a majority of cells demonstrated significant interactions among the cue type, spatial location, and relative position factors, indicating that the predominant correlate of hippocampal activity in this task involves specific conjunctions of, or relations among, multiple variables.

It is possible that the activity of cells related to conjunctions of spatial and nonspatial variables in the present study may reflect the activity of multiple neurons due to imperfect isolation during recording. However, the main findings of the present study do not depend on perfect unit isolation because the primary question we sought to answer concerns whether and to what extent nonspatial information is represented by the hippocampus when it is made salient and relevant to the task at hand. If multiple neurons contributed to some of the identified 

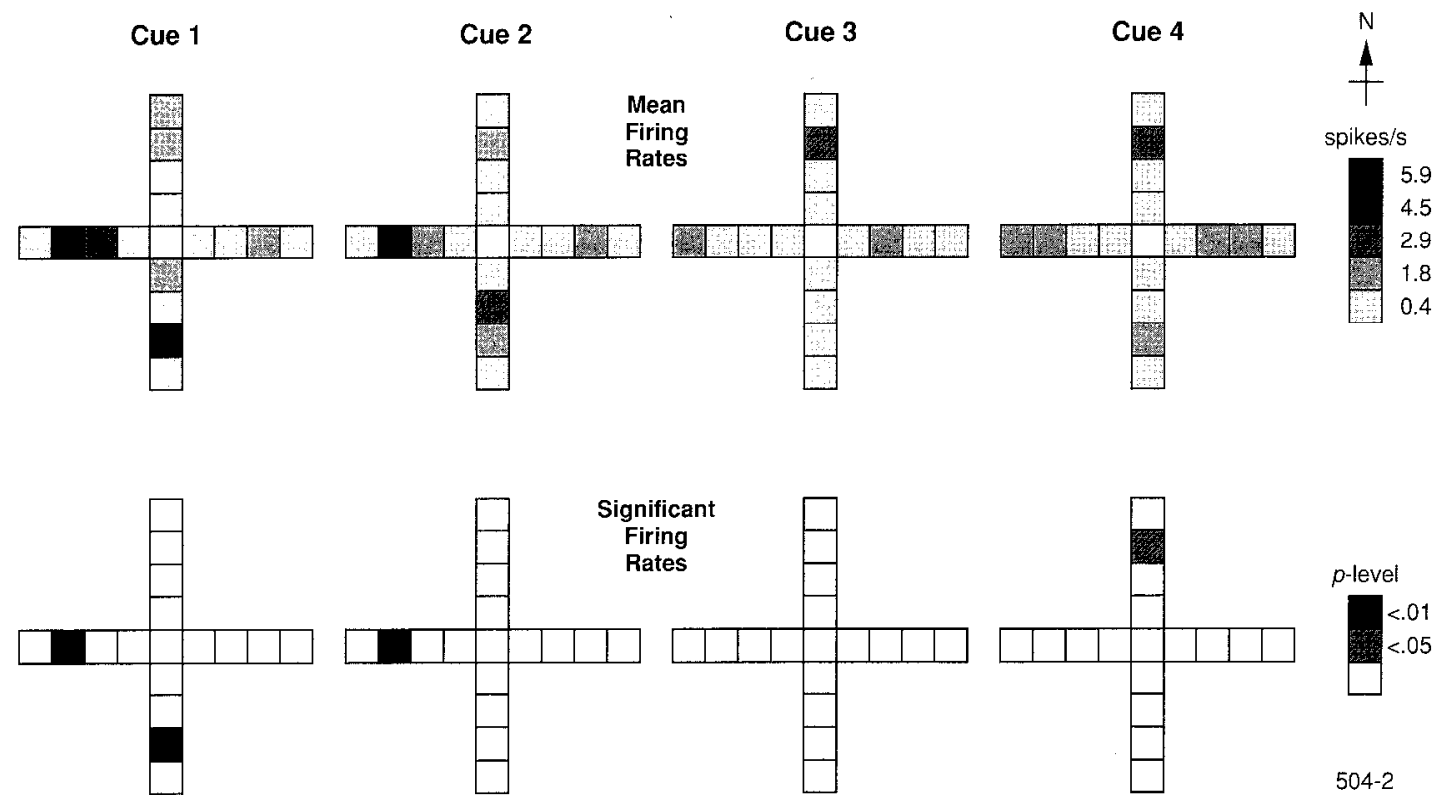

Figure 6. Example of a "relative position cell."

units, this would increase the likelihood of falsely observing conjunctions of the factors in single cells, but would not bias our conclusions about the extent to which spatial or nonspatial firing correlates were observed. Nevertheless, our unit isolation methods, using a combination of time and amplitude discrimination, are comparable to the most sensitive techniques that can be applied to extracellular recordings. Furthermore, given the prevalence of conjunctional properties of the unit firing correlates, it seems likely that, at least in many of our recordings, the observed interactions among nonspatial and spatial variables reflect the coding of relations between these factors by single hippocampal cells.

In our view, these findings support three general conclusions about the nature of hippocampal coding. First, in a situation where spatial information is not emphasized, hippocampal neuronal activity is strongly influenced by salient nonspatial cues and shows no particular preference for spatial representation. Second, comparing the present findings to those of other studies, it appcars the nature of hippocampal representation is altered corresponding to the stimuli and cognitive demands of different tasks in which the subject is engaged. Third, the fundamental and pervasive parameter encoded within hippocampal neural activity involves relationships among salient items and events. Each of these conclusions and their implications are discussed in the following sections.

\section{Nonspatial and spatial representation in individual hippocampal complex-spike cells}

The nonspatial task used in the present experiment is a somewhat unorthodox utilization of an apparatus that is more frequently used to study spatial learning. In the latter application, the intramaze environment is invariably uniform compared to a relatively rich extramaze environment. Hippocampal unit activity recorded in this type of situation usually correlates strongly with the location of the subject, resulting in well-defined "place fields" for most hippocampal complex-spike cells (e.g., O'Keefe and Dostrovsky, 1971; O'Keefe, 1976; Olton et al., 1978; Muller and Kubie, 1987). One possible interpretation of this finding is that hippocampal function is concerned with encoding the location of the animal in the apparatus. In this conceptualization, the array of extramaze stimuli are used by the subject to create a "cognitive map" of the environment for the purpose of navigation (O'Keefe and Nadel, 1978). However, an alternative explanation is that spatial correlates predominate because (1) the coding of spatial relations in the environment is essential to performance in these tasks, and (2) nonspatial information is minimized and made irrelevant. Consistent with this explanation, similar numbers of "cue cells" and "place cells" were obtained in the present study, and the significant effects obtained for these two cell types accounted for approximately equivalent amounts of the variance associated with the firing of individual units. Thus, when successful performance requires the use of salient local cues and distal spatial cues are minimized and made irrelevant, hippocampal activity displays no overwhelming representation of spatial cues.

The present findings are also consistent with and add to an increasing body of evidence indicating that the hippocampus is more broadly involved in the processing of stimuli than the cognitive mapping theory suggests. Nonspatial correlates of hippocampal unit activity have been described in conditioning and discrimination paradigms where spatial cues are not salient and are irrelevant to task performance. Thus, in the rabbit conditioned-eyeblink paradigm (Berger et al, 1983), delayed matching and nonmatching tasks (Wible et al., 1986; Otto and Eichenbaum, 1992; Hampson et al., 1993), and discrimination learning paradigms (Eichenbaum et al., 1987; Wiener et al., 1989), hippocampal cells fire in association with discrete nonspatial discriminative stimuli and learned responses. The present data indicate that prominent nonspatial correlates of hippocampal activity can be revealed even in "open-field" environments when nonspatial cues are salient and relevant.

Spatial location emerged as a prominent correlate of hippocampal activity in the present study despite our efforts to minimize extramaze cues in the testing environment. There are at least two possible sources from which the animal could obtain the information necessary to determine spatial location. First, 

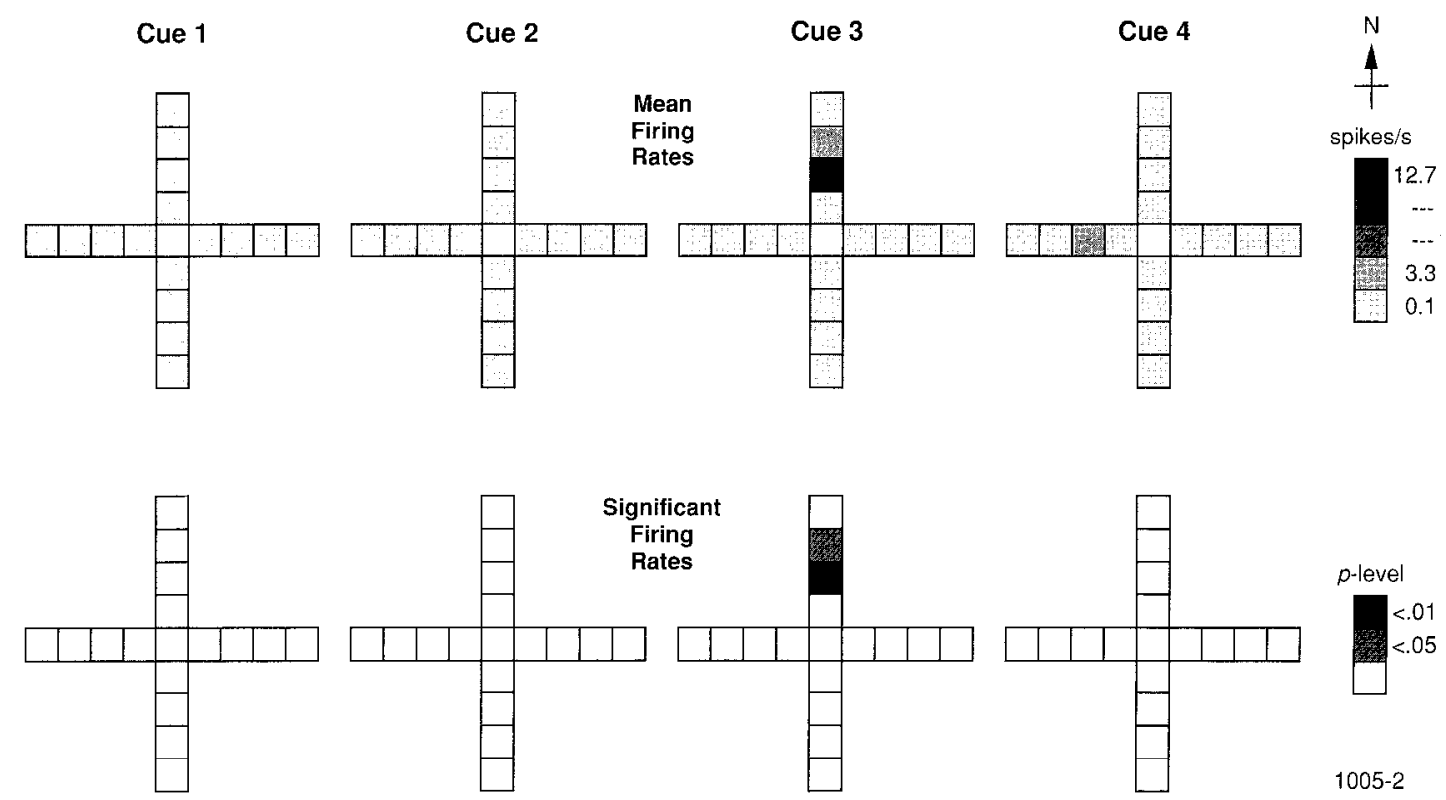

Figure 7. Example of an "interaction cell."

the animals might have been able to maintain a sense of orientation in the maze based on cues that were present only transiently. Place fields have been observed to persist even when the initial orienting cucs are removed (Muller and Kubic, 1987; O'Keefe and Speakman, 1987) or the experimental environment is darkened (Quirk et al., 1990), indicating that rats can maintain a sense of spatial orientation that is reflected in the hippocampal representation by a form of "dead reckoning." Furthermore, the cues necessary to support this sense of orientation can be quite fleeting. It has been shown that even the entry point into an ambiguous environment can provide sufficient cues for the animal to orient in space for several minutes (Sharp et al., 1990). Since such orienting cues were present in this experiment, it is not surprising that they were reflected in hippocampal cellular activity. A second source of information that might have allowed the animals to maintain a consistent spatial orientation is uncontrolled extramaze cues (e.g., the hum of the computer that was audible above the background masking noise; see Fig. 1). The influence of such cues is apparent even in tasks that involve rigid control of extramaze cues. O'Kecfe and Spcakman (1987) observed place fields related to uncontrolled and explicitly irrelevant spatial cues beyond the immediate screened-off testing area despite the presence of a set of orienting cues within the testing environment.

In addition, the present study confirms the observation of place fields even in situations where the animal is not required to use spatial relations to solve a behavioral task. Indeed, we observed approximately as many "place" cells as "cue cells," despite the explicitly nonspatial nature of the task. It should be noted, however, that in comparing the present findings with those from studies involving spatial tasks, we observed many fewer place cells in the present study. Thus, in rats performing spatial tasks, the activity of the overwhelming majority of complex-spikes cells is associated with distal spatial cues (e.g., O'Keefe and Conway, 1978; O'Keefe and Speakman, 1987), but in the present study no such predominance was observed. That spatial aspects of the environment were still represented by hippocampal firing properties even though spatial cues were minimized in the present study presumably reflects the likelihood that a sense of spatial awareness by the rat is always important and is captured by hippocampal cellular activity. In the present circumstances, then, both nonspatial and spatial regularities in the environment are important. Accordingly, the hippocampus performs "multitasking," representing both the relevant nonspatial and spatial relations in the environment. Nonspatial and spatial aspects of the task might have been encoded independently or, as suggested by the present data, might have been combined such that, even though the task did not require remembering spatial relations, the rat might have remembered previously visited arms in terms of their spatial locations on different trials.

Our suggestion that the development of place fields in the present experiment likely reflects the importance of a sense of consistent spatial orientation for the subject, regardless of its direct relevance to task performance, is supported by several previous observations on hippocampal place cells. Thus, several previous experiments have shown that hippocampal place fields develop even in open-field or maze environments that do not necessitate the use of available spatial cues. Hippocampal complex-spike cells exhibit well-developed place fields in tasks that involve simply retrieving food pellets scattered randomly around an arena (Muller and Kubie, 1987; Muller et al., 1987), and in radial maze tasks in which all trials involve "forced" entry into arms (McNaughton et al., 1983). This development of place fields, reflecting the encoding of relevant spatial relations in the environment, should, in our view, be expected in virtually any paradigm where the animal is unrestrained and given even minimal information allowing a sense of spatial orientation. While the learning of spatial regularities of the environment is "incidental" to the explicit task demands, it is possible the rat is motivated to keep track of its location in space independent of the task demands, or that the rat attempts to remember where it has visited different nonspatial cues or otherwise attempts to store the previous spatial configurations of the cues.

\section{Hippocampal complex-spike cell firing is determined by} task-relevant variables

One interpretation of the mixture of findings on spatial and nonspatial correlates of hippocampal unit activity is that the 
environmental cues and behaviors that are captured by hippocampal unit activity can change under different behavioral circumstances, and that the predominant hippocampal firing correlates may reflect the kinds of information that are most salient and relevant to the task at hand. A study by Wiener et al. (1989), in which rats performed both an odor discrimination task and a spatial memory task in the same environment, offers direct evidence in support of this view. During the performance of the odor discrimination task, the firing of many cells, including those that fired only when the rat was at a particular location, was time locked to individual trial events including the approach to, and sampling of, the relevant odor stimuli. However, during performance of the spatial task, no increased firing was observed at the locations where odor-task-related activity had been observed, and the same neurons displayed well-defined place fields at other loci. In addition, Qin et al. (1993) reported preliminary evidence showing that the firing qualities of place cells changed across two variations of an openfield task that involved identical spatial cues, but differed only in their behavioral requirements. These findings, combined with the present results, strongly suggest that the extent to which stimuli and events are reflected in hippocampal activity is influenced by the degree to which they are salient and relevant to the task at hand.

The discovery that the relative position of the animal can be an important variable determining hippocampal unit activity may be interpreted as consistent with the view that hippocampal neurons encode relationships among items and events that are particularly relevant to the task at hand. In the present study we sometimes interpreted a significant effect of relative position as a qualification or specification of a "cue" or "spatial location." For cells where there was no significant main effect or interaction with cue type or spatial location, a significant RP main effect may be interpreted as the encoding of a behavioral event that characteristically occurs at a particular position. This interpretation is consistent with the observation that the activity of hippocampal place cells is strongly modulated by the speed of movement, direction, and turning angle of the subject within a place field (McNaughton et al., 1983; Wiener et al., 1989). Alternatively, a relative position firing correlate may reflect the processing of relative spatial position per se. Notably, while the encoding of relative position clearly involves a "spatial" parameter, it cannot be construed as a "place field" becausc it does not represent a single location defined by allocentric cues. Rather, radial position constitutes a consistent relational variable for an instance of exch trial, and the high degree to which it was represented by hippocampal cellular activity may reflect its importance in encoding the stream of trial experiences.

\section{What is the fundamental coding property of hippocampal neurons?}

The activity of more than half of the cells that displayed significant firing correlates was related to both cue type and spatial location. This finding concurs with other results showing hippocampal neuronal activity often reflects interactions between spatial and nonspatial information in situations where there are clear spatial cues and relevant nonspatial stimuli. In a delayed match to sample task where rats were required to choose the appropriate goal box based on its color (Wible et al., 1986), the activity of almost one-third of the cells displaying significant effects was related to both the color and position of the goal arm. Similarly, Rolls et al. (1989), testing monkeys in a pattern matching task, found that some hippocampal neurons fired selectively when an individual pattern was displayed in a particular position on the choice panel, and Ono and colleagues (Tamura et al., 1990; Ono et al., 1991) have also observed visually driven hippocampal cells whose activity is dependent on the position of the animal in the environment as well as on the locations of the relevant visual cues. Wiener et al. (1989), using a simultaneous odor discrimination task, showed the activity of some hippocampal neurons was differentially associated with specific configurations of odors and their presentation positions. The present results also indicate that relative position may be incorporated into the conjunctions of items and events encoded by hippocampal activity. This finding is consistent with the data of Rolls, Ono, and their colleagues, showing that hippocampal neuronal activity reflects a variety of egocentric, as well as allocentric, spatial dimensions encoded along with specific cues in monkeys performing pattern discrimination tasks.

That the activity of more than a third of the units presented in Table 1 was associated with the cue type, spatial location, and relative position factors emphasizes the apparent role of the hippocampus in representing conjunctions of variables that are relevant to ongoing behavior. Indeed, the proportion of variance associated with the interaction effects of these units was consistently high for three-way interactions and, to a lesser extent, two-way interactions, suggesting that their principle function was conjunctional representation. This result, combined with the data cited above, further indicates that when certain firing correlates predominate (e.g., place) they may reflect more the constraints of the experimental design than the constraints of hippocampal representation.

The encoding of both spatial and nonspatial information by hippocampal neurons has important implications for the role of the hippocampus in mnemonic processing. Clearly, the present findings are inconsistent with accounts that strictly limit hippocampal function to spatial processing and, as such, are incompatible with O'Keefe and Nadel's (1978) "cognitive mapping" theory, which restricts the role of the hippocampus to the encoding of spatial information. The results indicate that the predominance of spatial coding observed in many experiments is more a reflection of the disproportionate emphasis placed on spatial cues in such paradigms than it is a comprehensive characterization of the fundamental property of hippocampal processing. The present data necessitate, at the least, a broader account in which spatial location may be an omnipresent component of stimulus coding but not the only parameter captured, nor even the most important parameter in some situations.

The present results are consistent with this notion and contribute to a growing body of evidence supporting the view that the hippocampus mediates a memory representation based on various relationships between multiple independent stimuli (Eichenbaum et al., 1992a,b; Cohen and Eichenbaum, 1993). According to this more general formulation, spatial learning is an excellent example of relational processing in which the hippocampus represents the topological relations between the multiple distal visual cues used to guide performance. In the present study, the animal was required to remember particular visits to maze arms identified by specific cues within the same session; thus, the task required the animal to encode temporal, rather than spatial, relations among particular cpisodes involving the nonspatial cues. Our conclusion based on the present findings is that, under circumstances where local nonspatial cues are emphasized, hippocampal cells encode such cues prominently 
in relation to spatial and other aspects of the task at hand. As observed in other experiments (Christian and Deadwyler, 1986; Wible et al., 1986; Eichenbaum et al., 1987; Otto and Eichenbaum, 1992), these aspects could also include the temporally defined sequence of events associated with this working memory task, as well as variables specifically examined here. Our conception of such relational processing by the hippocampus is that the encoding of conjunctions of items and events reflects the construction and modification of a network of representations supporting flexible "declarative" expression of memories.

\section{References}

Berger TW, Rinaldi PC, Weisz DJ, Thompson RF (1983) Single-unit analysis of different hippocampal cell types during classical conditioning of the nictitating membrane response. J Neurophysiol 50: 1197-1219.

Christian EP, Deadwyler SA (1986) Behavioral functions and hippocampal cell types: evidence for two nonoverlapping populations in the rat. J Neurophysiol 55:331-348.

Cohen NJ, Eichenbaum H (1993) Memory, amnesia, and the hippocampal system. Cambridge, MA: MIT Press.

Eichenbaum H, Perrijohn D, Deluca AM, Chorover SL (1977) Compact miniature microelectrode-telemetry system. Physiol Behav 18: 1175-1178.

Eichenbaum H, Kuperstein M, Fagan A, Nagode J (1987) Cue-sampling and goal-approach correlates of hippocampal unit activity in rats performing an odor discrimination task. J Neurosci 7:716-732.

Eichenbaum H, Cohen NJ, Otto T, Wible CG (1992a) Memory representation in the hippocampus: functional domain and functional organization. In: Memory: organization and locus of change (Squire LR, Lynch G, Weinberger NM, McGaugh JL, eds), pp 163-204. London: Oxford UP.

Eichenbaum H, Otto T, Cohen NJ (1992b) The hippocampus-what does it do? Behav Neural Biol 57:2-36.

Hampson RE, Heyser CJ, Deadwyler SA (1993) Hippocampal cell firing correlates of delayed-match-to-sample performance in the rat. Behav Neurosci 107:715-739.

Kubie JL (1984) A driveable bundle of microwires for collecting singleunit data from freely moving rats. Physiol Behav 32:115-118.

Kubie JL, Ranck JB (1983) Sensory-behavioral correlates in individual hippocampus neurons in threc situations: space and context. In: Neurobiology of the hippocampus (Siefert W, ed), pp 433-447. London: Academic.

McNaughton BL, Barnes CA, O'Keefe JA (1983) The contributions of position, direction, and velocity to single unit activity in the hippocampus. Exp Brain Res 52:41-49.

Muller RU, Kubie JL (1987) The effects of changes in the environment on the spatial firing of hippocampal complex-spike cells. J Neurosci 7:1951-1968.

Muller RU, Kubie JL, Ranck JB (1987) Spatial firing patterns of hippocampal complex spike cells in a fixed environment. J Neurosci $7: 1935-1950$

O'Keefe JA (1976) Place units in the hippocampus of the freely moving rat. Exp Neurol 51:78-109.

O'Keefe J, Conway DH (1978) Hippocampal place units in the freely moving rat: why they fire when they fire. Exp Brain Res 31:573-590.

O'Keefe J, Dostrovsky J (1971) The hippocampus as a cognitive map. Preliminary evidence from unit activity in freely moving rats. Brain Res 34:171-175.

O'Keefe J, Nadel L (1978) The hippocampus as a cognitive map. London: Oxford UP.

O'Keefe J, Speakman A (1987) Single unit activity in the rat hippocampus during a spatial memory task. Exp Brain Res 68:1-27.

Olton DS, Feustle WA (1981) Hippocampal function required for nonspatial working memory. Exp Brain Res 41:380-389.

Olton DS, Branch M, Best P (1978) Spatial correlates of hippocampal unit activity. Exp Neurol 58:387-409.

Ono T, Tamura R, Nakamura K (1991) The hippocampus and space: are there "place neurons" in the monkey hippocampus? Hippocampus 1:253-257.

Otto T, Eichenbaum H (1992) Neuronal activity in the hippocampus during delayed non-matching to sample performance in rats: evidence for hippocampal processing in recognition memory. Hippocampus 2:323-334.

Qin Y, Markus EJ, McNaughton BL, Barnes CA, Gothard KM (1993) Place field directionality: relation to visual, behavioral and spatial variables. Soc Neurosci Abstr 19:794.

Quirk GJ, Muller RU, Kubie JL (1990) The firing of hippocampal place cells in the dark depends on the rat's recent experience. J Neurusci 10:2008-2017.

Rolls ET, Miyashita Y, Cahusac P, Kesner RP, Niki HD, Feigenbaum JD, Bach L (1989) Hippocampal neurons in the monkey with activity related to the place where a stimulus is shown. J Neurosci 9:1835-1846.

Sharp PE, Kubie JL, Muller RU (1990) Firing properties of hippocampal neurons in a visually symmetrical stimulus environment: contributions of multiple sensory cues and mnemonic processes. J Neurosci 10:3093-3105.

Tamura R, Ono T, Fukuda M, Nakamura K (1990) Recognition of egocentric and allocentric visual and auditory space by neurons in the hippocampus of monkeys. Neurosci Lett 109:293-298.

Wible CG, Findling RL, Shapiro M, Lang EJ, Crane S, Olton DS (1986) Mnemonic correlates of unit activity in the hippocampus. Brain Res 399:97-110.

Wiener SI, Paul CA, Eichenbaum H (1989) Spatial and behavioral correlates of hippocampal neuronal activity. J Neurosci 9:2737-2763. 